\title{
Instantaneous Signal Collision Detection Using In-Band Full-Duplex: Machine Learning VS Domain-specific Knowledge
}

\author{
Seyed Ali Hassani ${ }^{1}$, Xianjun Jiao ${ }^{2}$, Ingrid Moerman ${ }^{2}$, Sofie Pollin ${ }^{1}$ \\ ${ }^{1}$ Department of Electrical Engineering, KU Leuven, Belgium \\ ${ }^{2}$ IDLab, imec - Gent University, Gent, Belgium
}

\begin{abstract}
Collision detection (CD) is a key capability of carrier sense multiple access (CSMA) based medium access control (MAC) protocol. Applying CD, the transmitter can abort transmission immediately so that the power can be saved. This technique does not need the peer receiver to give feedback on whether there is a packet collision, and hence, the overall overhead is significantly low. The challenge, however, is to operate in transmit time and instantly detect the week colliding signal in the presence of strong self-interference (SI). This paper investigates two CD methods. The first technique trains a convolutional neural network (CNN) model which operates on raw baseband samples, without the need for pre-decoding. The second method treats the SI as a normal signal and estimates the signal to noise ratio (SNR): low SNR implies there is a collision because the pure SI is expected to have high SNR. Both models are evaluated by IEEE 802.15.4-like measured and simulated signals. The results show that collisions up to $30 \mathrm{~dB}$ below the SI signal can be detected precisely within $20 \mu \mathrm{s}$, while the proposed models can deliver acceptably low false alarm rate $<1.5 \%$.
\end{abstract}

Index Terms-In-band full-duplex, self-interference cancellation, collision detection, convolutional neural network, SNR estimation.

\section{INTRODUCTION}

Thanks to the recent advances in wireless technology, we have witnessed remarkable improvement in wireless networking in such a way that today, a broad diversity of applications with particular requirements can be developed. Due to this successful performance, it is anticipated to end up with billions of networked devices which compete to access the communication medium [1]. Beside the fast-growing densification of wireless networks, the scarcity of spectrum highlights the necessity of efficient medium access control (MAC) protocols that can minimize the cost of packet collision and interference. It occurs in contention-based networks when two or more wireless nodes start transmission with overlap in time. In such a condition, the receiving node fails to decode the packet, leading retransmission and extra energy consumption [2].

In addition, a probable packet collision also can severely impact the latency performance and consequently degrades the reliability. It is, therefore, more crucial in applications such as vehicular networks, self-driving cars and tactile internet [3], where the network has to fulfill low-latency $(1 \mathrm{~m})$ and ultra-reliability $(99.99 \%)$ requirements. As a primary solution, carrier sense multiple access (CSMA) with collision avoidance

The first two authors contributed equally to this paper.
(CA) is introduced [4], [5]. This MAC scheme applies a random back-off mechanism to achieve distributed medium access. The performance of this mechanism, however, can be significantly reduced by growing the number of nodes [2]. Although prolonging the back-off delay may resolve the contention problem, yet it negatively affects the latency, reliability, and throughput. To avoid the long back-off delay in dense networks, the CSMA MAC protocol with collision detection (CD) is developed. Via an instantaneous collision detection, the node can take an appropriate reaction to lessens the collision's impact. For instance, the transmitter can abort the ongoing transmission to prevent the waste of power or vacate the channel for a high-priority message. Intensive research has been done to perform $\mathrm{CD}$ at the receiver side, mostly relying on a feedback signal to notify the transmitter. This approach either requires a high transmission power or demands a dedicated out-of-band control channel [6].

Instant $C D$ can be achieved at the transmitter side more efficiently. This approach resolves the need for a feedback signal and does not require any contribution from the receiver node. To detect the collision, though, the device has to overcome the strong self-interference (SI) in such a way that it can listen to the channel in transmit time. This capability is feasible by in-band full-duplex (IBFD) technology [7], [8], which allows two nodes to communicate concurrently over the same frequency channel. Recent works have shown the network-level benefits of using IBFD for collision detection [9]-[11], in the sense of power efficiency, throughput, and delay performance.

By any means, the performance of these MAC protocols highly depends on the rapidity and precision of the CD method, although the collision detection in this context has still not been investigated thoroughly. In [12], the authors study two different IBFD CD techniques: the energy detector, and the goodness-of-fit (GoF) test. The GoF test compares two empirical cumulative distribution functions (CDFs), belonging to the interfered and the pure SI signal, to judge whether they are in agreement. The measured results reveal that the Kuiper (KP) criteria [13] for the GoF test outperforms the energy detector, allowing nearly $100 \%$ accurate detection within $250 \mu \mathrm{s}$. Apart from its computational overhead, this statistical technique is not still fast enough to satisfy the mentioned applications. 


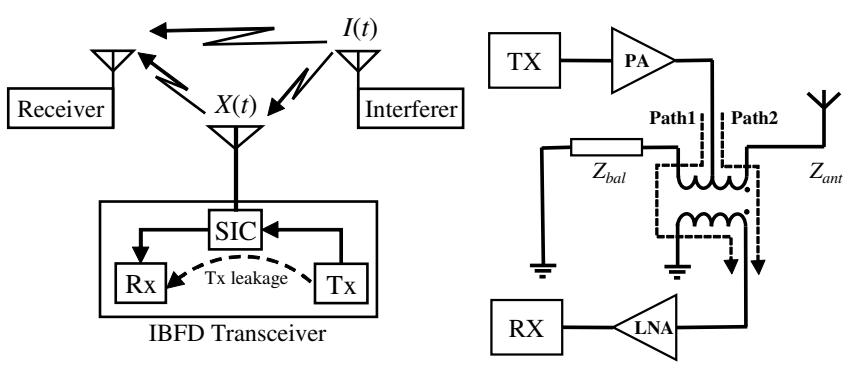

Fig. 1. (Left): System model overview. (Right): Analog self-interference cancellation using electrical balance duplexing.

By leveraging multiple hidden convolutional layers, modern machine learning techniques have confirmed their effectiveness in a wide range of applications, such as automatic image recognition, and network optimization. Motivated by its remarkable success in various fields as well as its realtime functionality, in this paper we investigate whether a convolutional neural network $(\mathrm{CNN})$ can be exploited to accelerate $\mathrm{CD}$ without sacrificing the detection accuracy.

Meanwhile, we realize that the CD problem can be mapped to traditional SNR estimation problem. When there is a collision, the signal SNR will drop. Lots of domain knowledge are there with regard to signal demodulation and SNR estimation. On the contrary, CNN could be regarded as a kind of domainspecific knowledge less method. It will be interesting to see the performance comparison between the two methodologies. This kind of comparison will inspire the research community to study further about how should we combine the domainspecific knowledge (DSK) with CNN. Besides, to encourage future studies, we offer free access to the data and programs in [14] and [15], which allows researchers to reproduce our results out of the box or investigate different approaches.

The rest of this paper is constructed as follows. Section II briefly introduces the concept of IBFD transmitter-based collision detection, including a brief overview of in-band selfinterference cancellation. The proposed CD approaches are described in Section III and Section IV. Section V represents the measurement setup and discusses the performance metrics. The results are then presented in Section VI and finally, conclusion is drawn in Section VII.

\section{IBFD TRANSMitTER-BASED COLlision DETECTION}

\section{A. System Model}

Fig. 1 (Left) illustrates the system model of the IBFD transmitter-based CD system. The model consists of an IBFD transceiver which is equipped with an analog self-interference cancellation (SIC) module, a receiver, and the interferer. While the transceiver sends a signal to the receiver, the interferer may cause packet collision as a result of missynchronization or contention. To detect an occurring packet collision, the transceiver has to solve a binary hypothesis test [12] based on the received signal at the transceiver node $Y(t)$ and decide on the correct hypothesis:

$$
Y(t)= \begin{cases}h_{x x}(t) * X(t)+W(t) & \text { if } \mathbb{H}_{x} \\ h_{x x}(t) * X(t)+W(t)+h_{x i}(t) * I(t) & \text { if } \mathbb{H}_{i}\end{cases}
$$

where $X(t)$ is the transmit signal by the transceiver, $h_{x x}(t)$ characterizes the SI channel, $h_{x i}(t)$ specifies the channel between the interferer and the IBFD transceiver, $I(t)$ denotes the signal transmitted by the interferer, $W(t)$ stands for the additive noise, and $(*)$ presents the time-domain convolution operator. The hypothesis $\mathbb{H}_{x}$ means that the received signal is merely the residual SI after analog SI rejection. Detection of the hypothesis $\mathbb{H}_{i}$ also implies that the IBFD transceiver is exposed to signal interference, and hence, it has to take an appropriate reaction to deal with it.

\section{B. Analog Self-interference Cancellation by Electrical Bal- ance Duplexing}

Channel-awareness in transmit time is not possible unless the undesired strong SI signal is suppressed sufficiently. Typically, an IBFD device comprises two stages of SI cancellation. The first provides the essential Tx-Rx isolation at the analog domain to enable further SI rejection at the digital sampled baseband. Fig. 1 (Right) depicts the structure of the electrical balance duplexer (EBD), which is an attractive analog SIC solution. As shown, the EBD consists of a hybrid transformer coupled with an impedance network $Z_{b a l}$. As long as the impedance network imitates the impedance of the antenna $Z_{\text {ant }}$, the two copies of the transmit signal (shown by dashed arrows in Fig. 1 (Right)) interfere destructively at the EBD's receiver port [16]. This can be done by a tuning algorithm which manipulates $Z_{b a l}$, aiming maximum Tx-Rx isolation. Due to the high level of Tx-Rx isolation that the EBD can provide, we employed it in this work.

\section{Collision Detection by a Multilayer Convolutional Neural Network}

Fundamentally, a CNN platform operates over the timedomain data stream, without the need for higher-level decoding or prerequisite advanced feature extraction. The model can learn multiple matched filters, so-called kernels, which enable extracting invisible patterns hidden in the data [17]. Depending on the size of the data-set and the classification complexity, the model requires relatively long learning time. However, once the model is trained, it can render instant identification which makes it suitable for the CD problem. This section represents the proposed CNN-based collision detector followed by the training details.

\section{A. Proposed CNN Architecture}

Aiming minimal network size, the designed CNN model comprises two successive convolutional layers, a single dense layer of 16 cells and the output layer, as shown in Fig. 2. The input to the network is a windowed sequence of $N$ amplitude/phase samples of the baseband received signal. The amplitude vector is $l^{2}$ normalized and the phase is also normalized between -1 and 1 . 


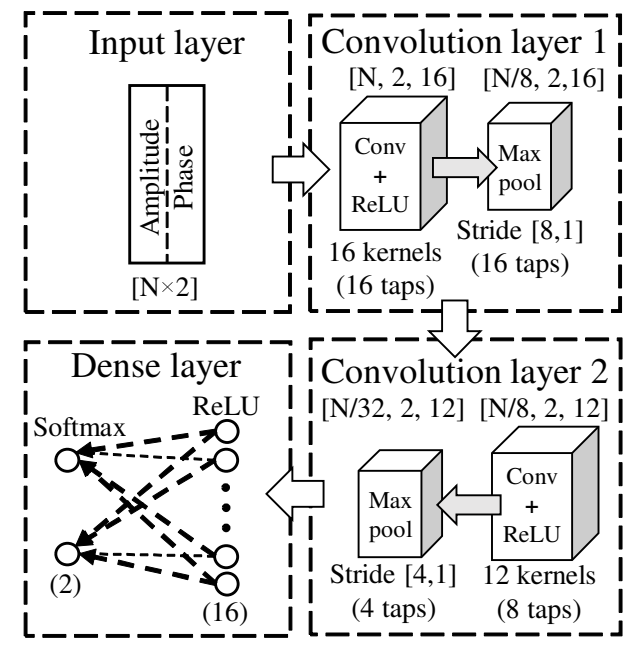

Fig. 2. Proposed $\mathrm{CNN}$ architecture used for signal collision detection.

The input vector is fed to the first convolution layer (CL), including 16 kernels, each comprises 16 taps. The resultant feature map is then passed through a rectified linear unit (ReLU) activation function [18] to introduce the non-linearity that is needed for learning the CD problem. The choice of this activation function was motivated by its optimum computational overhead. The ReLU operation can be mathematically described as

$$
\operatorname{ReLU}(x)=\max (0, x) .
$$

Typically, a max-pooling operator follows each of the convolutional layers. This block summarizes the outputs of neighboring groups of neurons in the same layer and reduces the dimensionality of the feature map to the most influential ones. The employed max-pool block in the first CL includes a sliding window of length 16 , which moves with a stride of 8 to prevent over-fitting during the training stage [19].

The second CL benefits from a similar structure, including 12 kernels of 8 taps and the ReLU operator, followed by a max-pool window of length 4 . The resultant output feeds the dense layer of the model, which contains 16 neurons with the ReLU activation function.

The output layer consists of two neurons that map the last feature map to one of the two ultimate classes, representing the hypothesis $\mathbb{H}_{x}$ and $\mathbb{H}_{i}$, introduced earlier in Sections II. In order to transform the non-normalized output of the network to a probability distribution over the predicted hypothesis, the softmax activation function is applied in this layer. The softmax operator can be presented as

$$
p\left(x_{i}\right)=\frac{e^{x_{i}}}{\sum_{j=0}^{1} e^{x_{j}}},
$$

where $x_{i}$ denotes the output of the neurons in the last layer whose softmax value is indicated by $p\left(x_{i}\right)$. Note that while the softmax operator aids the convergence of the model in the

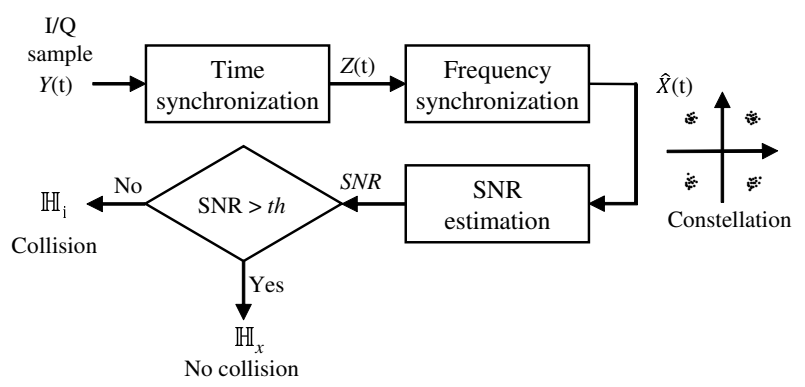

Fig. 3. SNR estimation based collision detection model overview.

training phase, it can be substituted with a ReLU activation function in the identification stage to reduce the computational overhead.

To train the model, the Adam algorithm [20], a first-order gradient-based optimizer with a learning rate of 0.0001 is employed. The aim is to minimize the softmax cross-entropy loss of $L$ defined as follows.

$$
L=-\sum_{i=0}^{1} y_{i} \log \left(p\left(x_{i}\right)\right),
$$

where $y_{i} \in\left\{y_{0}, y_{1}\right\}$ stands for the ground-truth collision detection probability, defined as follows.

$$
y= \begin{cases}\{0,1\} & \text { if } \mathbb{H}_{x} \\ \{1,0\} & \text { if } \mathbb{H}_{i}\end{cases}
$$

\section{Collision Detection Based on Domain-Specific KNOWLEDGE}

According to the signal model in (1), the main component $h_{x x}(t) * X(t)$ in the received signal $Y(t)$ is from the selftransmitted signal $X(t)$. In the setup of this paper, we know that $X(t)$ uses QPSK modulation, and $h_{x x}(t) * X(t)$ is much stronger than both noise $W(t)$ and interference $h_{x i}(t) * I(t)$. So we can use traditional communication signal processing algorithm to recover constellation of $X(t)$ and estimate SNR. When interference is not presented, SNR will be decided by both noise $W(t)$ and distortion function $h_{x x}(t)$, that reflect the hardware quality. When there is an interference, SNR will drop because the term $h_{x i}(t) * I(t)$ increases the noise floor. Here we assume $h_{x x}(t)$ is a scalar. Only timing and frequency error are considered, while other non-linear and frequency selectivity effects are ignored. In the topic of this paper, the collision causing SNR drop is our domain-specific knowledge.

The principle of SNR based collision detection is shown in Fig. 3. The appropriate algorithm is chosen according to the waveform characteristics and the hardware capability, such as clock accuracy $\varepsilon(p p m)$. The accumulated normalized sampling phase variation $\Delta \varphi_{s}$ during $N$ successive samples is

$$
\Delta \varphi_{s}=N \varepsilon
$$


Similarly, the carrier phase changes $\Delta \varphi_{c}$ can be written in the form

$$
\Delta \varphi_{c}=\frac{2 \pi N f_{c} \varepsilon}{f_{s}},
$$

where $f_{s}$ denotes the baseband sampling rate and $f_{c}$ is the carrier frequency.

Given the hardware features and the model parameters, in Section $V$ we show that $\Delta \varphi_{s}$ is relatively small and can be neglected regarding the SNR of QPSK signal. The carrier phase changing $\Delta \varphi_{c}$ during the input period is, however, more significant. Therefore, both carrier frequency and phase will be estimated and corrected.

\section{A. Algorithm Description}

Eq. (8) shows how the optimal sampling phase $p$ is decided.

$$
\underset{p \in[-1,1)}{\arg \max } S \hat{N} R\left(S Y N_{f}\left(f_{\text {int }}(Y(t), p)\right)\right),
$$

where $f_{\text {int }}(Y(t), p)$ means re-sampling the input signal $Y(t)$ with normalized relative sampling offset $p$ by specific interpolation method, such as linear or Makima [21]. $S Y N_{f}(Z(t))$ performs carrier synchronization on previously re-sampled signal $Z(t)=f_{\text {int }}(Y(t), p)$. To estimate frequency and phase of $Z(t)$, the 1st step is QPSK modulation removal by 4 times multiplication [22]: $Q(t)=Z(t)^{4}$.

Then $Q(t)$ only contains the phase rotation caused by frequency offset. The normalized phase and frequency estimation can be done based on (9) and (10), where 4 comes from four times multiplication, $N$ is the number of samples in $Q(t)$, $\arg ()$ calculates the phase of a complex sample.

$$
\begin{gathered}
\varphi_{\text {err }}=\left[\arg \sum_{i=0}^{N-1} Q\left(t_{i}\right)\right] / 4 \\
f_{\text {err }}=\frac{\arg \sum_{i=0}^{N / 2-1} Q\left(t_{i}\right)-\arg \sum_{i=N / 2}^{N-1} Q\left(t_{i}\right)}{4 N / 2}
\end{gathered}
$$

Frequency and phase correction to $Z(t)$ can be done by applying those estimations. The correction finally generates the estimation of $X(t)$, noted as $\widehat{X}(t)$, for SNR estimation. For QPSK modulation, SNR can be estimated per quadrant by (11) and (12) as in [23]:

$$
\begin{aligned}
S N R_{q} & =\frac{\left\|A_{q}\right\|^{2}}{\sum_{i \in \text { quadrant_q }}\left\|\left(\widehat{X}\left(t_{i}\right)-A_{q}\right)\right\|^{2} / N_{q}}, \\
A_{q} & =\sum_{i \in \text { quadrant_q }} \widehat{X}\left(t_{i}\right) / N_{q}, q \in\{0,1,2,3\},
\end{aligned}
$$

where $q$ is the quadrant index, $i \in$ quadrant_q means the sample index of all $\widehat{X}\left(t_{i}\right)$ falling into quadrant $q$, and $N_{q}$ is the number of samples in quadrant $q$. The overall SNR is given by the weighted SNR of each quadrant as follows.

$$
S N R=\sum_{q=0}^{3} \frac{S N R_{q} N_{q}}{N}
$$

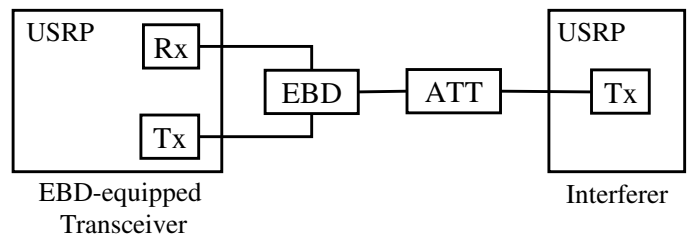

Fig. 4. Measurement setup used for generating the training and test databases.

\section{B. Optimum SNR threshold}

To decide the optimal SNR threshold for $\mathrm{CD}$, multiple random tests are performed based on I/Q samples with and without collision. Then we can have two approximate probability density functions (PDF) of SNR with and without collision $\mathbb{P}_{\mathbb{H}_{i}}(S N R), \mathbb{P}_{\mathbb{H}_{x}}(S N R)$. Based on the two PDFs, the optimal SNR threshold can be decided as

$$
\underset{t h}{\arg \min }\left[\mathbb{P}\left(S N R>t h \mid \mathbb{H}_{i}\right)+\mathbb{P}\left(S N R<t h \mid \mathbb{H}_{x}\right)\right] .
$$

Since we do not have analytic form PDF of SNR, above threshold can be calculated by numerical method. Because the interference is much weaker than self-interference, in practice SI level can be estimated easily from the amplitude of I/Q sample, but it is challenging to estimate interference level. So, different SI level may have various thresholds, but for a single SI level, all interference levels should share the same threshold.

\section{Measurement Setup and Performance Metrics}

We study the performance of the proposed CD techniques, using the baseband samples collected from an experimental setup, shown in Fig. 4. Similar to the used measurement setup in [12], the hardware installation combines two National Instruments USRPs, one of which is equipped with an EBD [16] to render the essential SI rejection at the analog domain. The other USRP plays the role of the interferer, by connecting its RF front-end to the EBD's antenna port via a variable attenuator. This arrangement allows for various path loss emulation. Both USRPs transmit the same IEEE 802.15.4-like signals at $0 \mathrm{dBm}$, and the measured noise power is $-90 \mathrm{dBm}$. The communication signal is four times oversampled both at the transmitter and at the receiver. The baseband sampled signal is recorded at $8 \mathrm{MHz}$ by the receiver of the EBDequipped USRP to be exploited for training and assessment of the proposed collision detectors. In order to tune the EBD, the particle swarm optimizer described in [24] is implemented on a MicroBlaze, which itself is deployed on the FPGA of the USRP. With this hardware configuration, one can achieve up to $60 \mathrm{~dB}$ SI rejection at the analog domain.

Given the carrier frequency at $f_{c}=2.4 \mathrm{GHz}$, worst case USRP's clock accuracy $\varepsilon=5 \mathrm{ppm}$ (official accuracy is $2.5 \mathrm{ppm}$ in data sheet of [25]), baseband sampling rate at $f_{s}=8 M S P S$, and employing $N=160$ samples for CD, one can estimate the accumulated carrier phase changes $\Delta \varphi_{s}$ and normalized sampling phase variation $\Delta \varphi_{s}, 1.5 \mathrm{rad}$ and 0.0008 
respectively. This validates the assumption in Section IV that the sampling phase variation has an insignificant impact on the CD problem.

To assess the proposed detector, we adopt the probability of correct collision/interference detection $\mathbb{P}_{\mathrm{D}}$ as a performance metric. Basically, $\mathbb{P}_{\mathrm{D}}$ is desired to be $100 \%$, which implies that the $\mathrm{CD}$ algorithm detects all occurring packet collisions. Although this metric can reflect the success rate of the correct detection, it does not entirely reveal the merit of the method.

Practically, the rate of incorrect detection is also equally important as it can severely affect the latency performance. For instance, the congestion window parameter should be double as determined by the binary-exponential back-off algorithm used in IEEE 802.11 [26]. Hence, we also acknowledge the probability of false alarm $\mathbb{P}_{\mathrm{F}}$ (incorrect collision detection) as the second intended performance metric which has to be minimized by the $\mathrm{CD}$ algorithm.

\section{RESULTS}

In addition to the measured database, which is detailed in Section V, the ideal IEEE 802.15.4 waveform is also simulated by MATLAB. This allows unbiased assessment, avoiding the impact of practical hardware imperfections, such as noise uncertainty, transmitter nonlinearity, etc. The databases comprise pure SI and interfered SI signals, assuming $40 \mathrm{~dB}$ and $60 \mathrm{~dB}$ analog SI rejection. We implemented the CNN platform using TensorFlow [27], which is a data flow graph based numerical computation library from Google. The model is then trained by a Nvidia GeForce GTX 1080 graphics card. For DSK-based $\mathrm{CD}$, the optimal SNR threshold is $34 d B$, that is optimized under $-70 \mathrm{dBm}$ interference, when SI cancellation is $40 \mathrm{~dB}$. Accordingly, the optimal threshold is $20 d B$ under the case of SI $60 \mathrm{~dB}$ Tx-Rx isolation and interference level at $-80 \mathrm{dBm}$. The CD performance is evaluated for 10240 instances of $N=160 \mathrm{I} / \mathrm{Q}$ samples $(20 \mu \mathrm{s})$.

Fig. 5 show the detection performance when the analog SIC provides $40 d B$ SI rejection. As shown in Fig. 5 (Up), both CD models can deliver nearly $100 \%$ correct detection even for the case the colliding signal is $30 d B$ below the residual SI signal. This graph also shows that different detection rates are achieved when the collision is more than $30 \mathrm{~dB}$ weaker than the residual SI. The reason lies in the fact that not all the hardware characteristics are contributed to generate the simulated database. Nevertheless, the CNN model produces more accurate detection in such a condition. Fig. 5 (Down) depicts the $\mathrm{CD}$ false alarm rate $\mathbb{P}_{\mathrm{F}}$. As shown, $\mathbb{P}_{\mathrm{F}}$ better than $1 \%$ is obtained in all cases.

To study the impact of the Tx-Rx isolation, the test is also repeated for $60 \mathrm{~dB}$ analog SI cancellation. As depicted in Fig. 6 (Up), in this case, the DSK model archives a better detection performance for the collisions $15 d B$ under the remaining SI. However, the false alarm rate $\mathbb{P}_{\mathrm{F}}$ is slightly better for the CNN modle, as shown in Fig. 6 (Down).

The reason behind the above results needs to be researched in the future work. A possible explanation is that the stronger
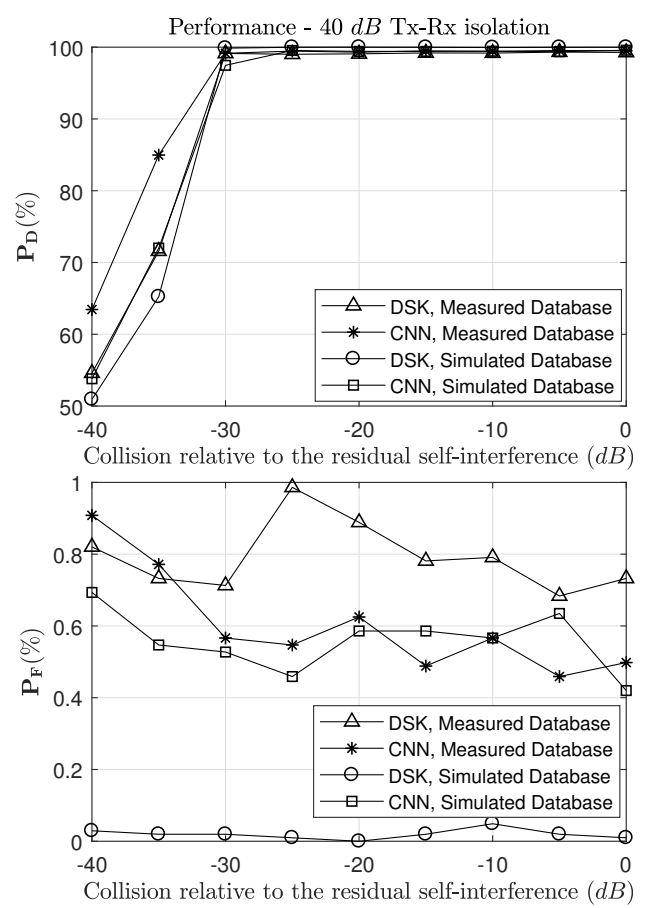

Fig. 5. Probability of collision detection $\mathbb{P}_{\mathrm{D}}(\mathrm{Up}) /$ false alarm $\mathbb{P}_{\mathrm{F}}$ (Down) VS the power of collision relative to the residual self-interference, when the analog Tx-Rx isolation is $40 \mathrm{~dB}$.
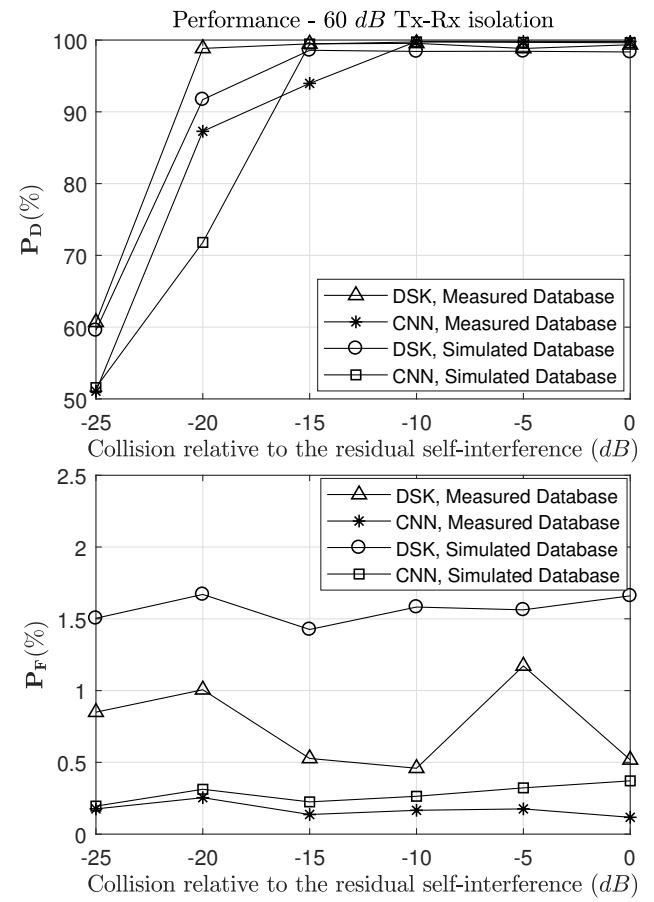

Fig. 6. Probability of collision detection $\mathbb{P}_{\mathrm{D}}(\mathrm{Up})$ / false alarm $\mathbb{P}_{\mathrm{F}}$ (Down) VS the power of collision relative to the residual self-interference, when the analog Tx-Rx isolation is $60 \mathrm{~dB}$. 
SI involves more non-ideal RF characteristics, such as nonlinear channel response, higher noise floor or phase noise. $\mathrm{CNN}$ somehow learns these characteristics and its performance is slightly higher in this case. The SNR based method always assumes the ideal linear signal model, so it performs slightly better in the weak SI case. The trade-off between performance on specific model and generality needs to be considered in the future, no matter machine learning or domain-specific knowledge is used.

\section{CONCLUSION}

This paper proposed machine learning and domain knowledge as two potential solutions to enhance signal collision detection (CD) in wireless IBFD CSMA/CD MAC protocol. Particularly, we developed a convolutional neural network (CNN) platform and a model based on domain-specific knowledge (DSK) to perform instantaneous CD. Both models are evaluated by measured and simulated datasets, considering $40 d B$ and $60 d B$ Tx-Rx isolation. The results show that the detection success depends on the extent of Tx-Rx isolation as well as the strength of the interfering signal.

Both CNN and DSK-based CD models could recognize nearly $100 \%$ of the collisions that are up to $30 d B$ below the remaining self-interference (SI) signal, with considerably low false alarm rate (1\%). The detection time is $20 \mu \mathrm{s}$, which is twelve-fold faster than the goodness-of-fit test proposed in [12]. We also conclude that the CNN model achieves a better detection sensitivity, if the remaining SI signal is not fully attenuated by the analog SI canceler. Otherwise, the DSKbased $\mathrm{CD}$ algorithm gives better detection sensitivity while increases the false alarm rate slightly.

\section{ACKNOWLEDGMENT}

This work was funded by the European Union's Horizon 2020 under grant agreement no. 732174 (ORCA project). We also would like to acknowledge Tom Vermeulen for providing us with the measured dataset and for his helpful comments.

\section{REFERENCES}

[1] L. Da Xu, W. He, and S. Li, "Internet of things in industries: A survey," IEEE Transactions on industrial informatics, vol. 10, no. 4, pp. 2233 2243, 2014

[2] S. Pollin, M. Ergen, S. C. Ergen, B. Bougard, L. Van der Perre, I. Moerman, A. Bahai, P. Varaiya, and F. Catthoor, "Performance analysis of slotted carrier sense ieee 802.15. 4 medium access layer," IEEE Transactions on wireless communications, vol. 7, no. 9, pp. 33593371, 2008.

[3] G. P. Fettweis, "The tactile internet: Applications and challenges," IEEE Vehicular Technology Magazine, vol. 9, no. 1, pp. 64-70, 2014.

[4] IEEE, "IEEE standard for local and metropolitan area networks part 15.4: Low-rate wireless personal area networks (LR-WPANs," IEEE 802.15.4-2011, 2011

[5] IEEE, "IEEE standard for information technology-part 11: Wireless LAN medium access control (MAC) and physical layer (PHY) specifications," IEEE 802.11-2012, 2012.

[6] S. Sen, R. R. Choudhury, and S. Nelakuditi, "CSMA/CN: Carrier sense multiple access with collision notification," IEEE/ACM Transactions on Networking (ToN), vol. 20, no. 2, pp. 544-556, 2012.

[7] A. Sabharwal, P. Schniter, D. Guo, D. W. Bliss, S. Rangarajan, and R. Wichman, "In-band full-duplex wireless: Challenges and opportunities," IEEE Journal on selected areas in communications, vol. 32, no. 9, pp. 1637-1652, 2014
[8] D. Kim, H. Lee, and D. Hong, "A survey of in-band full-duplex transmission: From the perspective of phy and mac layers," IEEE Communications Surveys \& Tutorials, vol. 17, no. 4, pp. 2017-2046, 2015.

[9] T. Vermeulen and S. Pollin, "Energy-delay analysis of full duplex wireless communication for sensor networks," in 2014 IEEE Global Communications Conference. IEEE, 2014, pp. 455-460.

[10] T. Vermeulen, F. Rosas, M. Verhelst, and S. Pollin, "Performance analysis of in-band full duplex collision and interference detection in dense networks," in 2016 13th IEEE Annual Consumer Communications \& Networking Conference (CCNC). IEEE, 2016, pp. 595-601.

[11] L. Song, Y. Liao, K. Bian, L. Song, and Z. Han, "Cross-layer protocol design for csma/cd in full-duplex wifi networks," IEEE Communications Letters, vol. 20, no. 4, pp. 792-795, 2016.

[12] T. Vermeulen, M. Laghate, G. Hattab, D. Cabric, and S. Pollin, "Towards instantaneous collision and interference detection using in-band full duplex," in IEEE INFOCOM 2017-IEEE Conference on Computer Communications. IEEE, 2017, pp. 1-9.

[13] N. H. Kuiper, "Tests concerning random points on a circle," in Nederl. Akad. Wetensch. Proc. Ser. A, vol. 63, no. 1, 1960, pp. 38-47.

[14] ORCA Project. (2019) Instantaneous IBFD signal CD: Machine learning VS domain-specific knowledge. [Online]. Available: https://github.com/orca-project/CD_DSK_CNN

[15] S. A. Hassani, X. Jiao, I. Moerman, and S. Pollin, "Instantaneous Signal Collision Detection Using In-Band Full-Duplex: Machine Learning VS Domain-specific Knowledge," in IEEE Dataport, 2020. [Online]. Available: http://dx.doi.org/10.21227/m9dg-pb95

[16] B. van Liempd, B. Hershberg, S. Ariumi, K. Raczkowski, K.-F. Bink, U. Karthaus, E. Martens, P. Wambacq, and J. Craninckx, "A +70-dBm IIP3 electrical-balance duplexer for highly integrated tunable front-ends," IEEE Transactions on Microwave Theory and Techniques, vol. 64, no. 12, pp. 4274-4286, 2016.

[17] S. Rajendran, W. Meert, D. Giustiniano, V. Lenders, and S. Pollin, "Deep learning models for wireless signal classification with distributed lowcost spectrum sensors," IEEE Transactions on Cognitive Communications and Networking, vol. 4, no. 3, pp. 433-445, 2018.

[18] V. Nair and G. E. Hinton, "Rectified linear units improve restricted boltzmann machines," in Proceedings of the 27th international conference on machine learning (ICML-10), 2010, pp. 807-814.

[19] B. Graham, "Fractional max-pooling," arXiv preprint arXiv:1412.6071, 2014.

[20] D. P. Kingma and J. Ba, "Adam: A method for stochastic optimization," arXiv preprint arXiv:1412.6980, 2014.

[21] C. Moler. (2019) Makima piecewise cubic interpolation. [Online]. Available: https://blogs.mathworks.com/cleve/2019/04/29/makima-piecewisecubic-interpolation/

[22] J. G. Proakis and M. Salehi, Digital communications. McGraw-hill New York, 2001, vol. 4.

[23] M. D. McKinley, K. A. Remley, M. Myslinski, J. S. Kenney, D. Schreurs, and B. Nauwelaers, "EVM calculation for broadband modulated signals," in 64th ARFTG Conf. Dig, 2004, pp. 45-52.

[24] T. Vermeulen, B. van Liempd, B. Hershberg, and S. Pollin, "Realtime rf self-interference cancellation for in-band full duplex," in 2015 IEEE International Symposium on Dynamic Spectrum Access Networks (DySPAN). IEEE, 2015, pp. 275-276.

[25] E. Research. (2014) Usrp x300. [Online]. Available: http://www.ettus.com/wpcontent/uploads/2018/11/X300_X310_Spec_Sheet.pdf

[26] S. Rayanchu, A. Mishra, D. Agrawal, S. Saha, and S. Banerjee, "Diagnosing wireless packet losses in 802.11: Separating collision from weak signal," in IEEE INFOCOM 2008-The 27th Conference on Computer Communications. IEEE, 2008, pp. 735-743.

[27] M. Abadi, A. Agarwal, P. Barham, E. Brevdo, Z. Chen, C. Citro, G. S. Corrado, A. Davis, J. Dean, M. Devin et al., "Tensorflow: Large-scale machine learning on heterogeneous distributed systems," arXiv preprint arXiv:1603.04467, 2016. 